\title{
A Survey on Routing Protocols in Mobile Ad Hoc Networks
}

\author{
N.Nithya ${ }^{1}$, R.Maruthaveni ${ }^{2}$ \\ ${ }^{1}$ Research Scholar, Department of Computer Science, \\ Dr. SNS Rajalakshmi College of Arts \& Science, Coimbatore, India \\ nithi5nimmi@gmail.com \\ ${ }^{2}$ Assistant Professor, Department of Computer Science, \\ Dr. SNS Rajalakshmi College of Arts \& Science, Coimbatore, India \\ dhanu_vene@gmail.com
}

\begin{abstract}
The main objective of this paper is to investigate the environment based protocol such as AODV, AOMDV and LCR .Mobile Ad hoc networks can be also defined as a collection of mobile nodes that intercommunicate on shared wireless channels. The primary goal of such an ad hoc network routing protocol is to provide correct and efficient route establishment between pair of nodes so that messages may be delivered in time. From this survey paper we can conclude that the AOMDV protocol performs better than the other two protocols in an Ad hoc Network.
\end{abstract}

Keywords: Ad hoc Networks, routing protocols, clustering, wireless networks, AODV, AOMDV, LCR.

\section{Introduction}

When Mobile Ad hoc Network (MANET) [1] is a collection of mobile devices and it is a self configuring. Routing in networking is the process of selecting the topology of the network. Devices in mobile ad-hoc networks should be able to detect the presence of other devices and perform the necessary set-up to facilitate communications and the sharing of data and services. changes with time due to mobility of nodes paths in a network to send network traffic Routing protocols in MANETs can be classified as Proactive (Table driven), Reactive (On demand) and Hybrid.

A proactive protocol, every node maintains the network topology information in the form of routing tables by periodically exchanging the Network In the proactive routing scheme each node maintains one or more tables to store consistent and up-to-date routing information from one to every other node routing information in the entire Networks. DSDV

A Reactive protocol, do not exchange routing information periodically. Here the paths are maintained only if the network needs it. It is done only through the route discovery process .It includes some of the routing protocols such as DSR, AODV, AOMDV.

A Hybrid protocol, as the name indicates it includes both the action of proactive and reactive protocol. Only a local network consisting of several neighboring nodes and it include the route discovery path for the network nodes that are used in the process, ZRP.

Layered Cluster based routing protocol, LCR is the clustering process that involves the cluster techniques .The process of dividing the network into interconnected substructures is called clustering and the interconnected substructures are called clusters. The Cluster based routing provides an answer to address nodes heterogeneity, and to limit the amount of routing information that propagates inside the network. The main advantage of lcr is that the nodes in the clusters are smaller to communicate when comparing to the entire networks.

Ad hoc on demand distance vector routing protocol,[2] $A O D V$ is an single path routing protocol. AODV relies on dynamically establishing route table entries at intermediate. Nodes in AODV the routing table is expanded by a sequence number to every destination and by time to live for every entry. Route discovery cycle that is used for route finding Provides uni cast and multicast Communication.

Ad hoc on demand multipath distance vector routing protocol [3], AOMDV is an multi path routing protocol AOMDV utilizes its multipath functionality at each hop, while DSR-MP provides its multi-path facility only at the source node, with minimal support for en-route re-direction. This enables AOMDV to use alternate routes to support the ongoing connections and thus lowers the packet drop rate. In cases where no alternate paths are available at an intermediary node and no routes can be discovered in the maximum route discovery interval, packets are simply dropped.

\section{Related Works}

In this section we present some of existing works on survey of routing protocols in MANETs.

[1]In C. E. Perkins and E. M. Royer proposed that the single-path routing protocols, route maintenance may be performed after route fail. Therefore, data transmission will be stopped while the new route is established, causing data transmission delay.

[2] Mahesh K. Marina and Samir R. das proposed that the Multipath routing establishes multiple routes between source and destination nodes. For fault tolerance, even if one route failure occurs, source nodes can maintain 
connections by using other routes. So multiple routing protocols can reduce data transmission failures and delay times that are caused by route disconnection.

[3] Multipath routing can be used in on-demand protocols to achieve faster and efficient recovery from route failures in highly dynamic ad hoc networks. In this paper, we have proposed an on-demand multipath distance vector protocol AOMDV that extends the single path AODV protocol to compute multiple paths. There are two main contributions of this work:

1. We show how route discovery mechanisms in the AODV protocol can be modified to obtain link disjoint multiple paths from source and intermediate nodes to the destination.

2. We use the notion of an advertised hop count to maintain multiple loop free paths in the distance vector.

In [5] A. Bhatnagar and T. G. Robertaz proposed the LCR which is the multipath routing algorithm using multi-level cluster structure to improve scalability. Besides, it transfers the Route Discovery procedure to the 2-server level to prevent the network flooding due to the LCR and DSR Route Discovery. Thus, Route Discovery does not require flooding mechanism and overhead is minimized. It can solve the path reliability problem by providing soft QoS guarantees with respect to end-to-end reliability by discovering a set of multiple disjoint paths and transmitting data along these paths.

\section{Survey on Three Routing Protocols}

3.1 Ad hoc on demand distance vector routing protocol,[2] $A O D V$, which is used to provide secure and reliable data transmission over the MANETs [6]. AODV discovers a route through network wide road casting. The source host starts a route discovery by broadcasting a route request to its neighbors. In the route request, there is a requested destination sequence number which is 1 greater than the destination sequence number currently known to the source.

[1] In C. E. Perkins and E. M. Royer, have presented a distance vector algorithm that is suitable for use with ad hoc networks AODV avoids problems with previous proposals notably DSDV and has the following features. Nodes store only the routes that are needed, Need for broadcast is minimized, Reduces memory requirements and needless duplications.

In AODV [7] Zahian Ismail, Rosilah Hassan 2011, proposed that the node uses hello messages to maintain the connectivity of neighboring nodes. Therefore, the link status to the next hop in an active route can be monitored. When a node discovers a link disconnection, it broadcasts a route error (RERR) packet to its neighbors, which in turn propagates the RERR packet towards nodes whose routes may be affected by the disconnected link. Then, the affected source can re-initiate a route discovery operation if the route is still needed.

In AODV [8], Misra, R.; Manda, C.R described each node maintains a cache to keep track of RREQs it has received. The cache also stores the path back to each RREQ originator. When the destination or a node that has a route to the destination receives the RREQ, it checks the destination sequence numbers it currently knows and the one specified in the RREQ.

In AODV, [11] single-path routing protocols, route maintenance may be performed after route fail. Therefore, data transmission will be stopped while the new route is established, causing data transmission delay. On the other hand, multipath routing protocols perform the route maintenance process even if only one route fails among the multiple routes. To perform the route maintenance process before all routes fail, the network must always maintain multiple routes. This can reduce data transmission delays caused by link failure].

In [10], Yu-Chee Tseng, Wen-Hua Liao, Shih-Lin Wuhave proposed that the Ad-hoc routing, when a route is needed to some destination, the protocol starts route discovery. Then the source node sends route request (RREQ) message to its neighbors, if those nodes do not have any information about the destination node, then they send the message to all its neighbors and so on, if any neighbor node has the information about the destination node, the node sends route reply message to the route request message initiator.

In AODV [12], D. Xu, M. Chiang, and J. Rexford the performance loss of joint congestion control and routing when routing is restricted to single-path routing as compared to the case where users can use multiple paths. We demonstrate that the total number of paths needed to achieve the optimal multipath utility is no greater than the sum of the number of links and the number of users. Furthermore, the average performance loss diminishes as the number of users tends to infinity.

\section{Advantages and Disadvantages of the AODV Routing Protocol}

The main advantage of this protocol is that routes are established on demand and destination sequence number is used to find the latest route to destination .The main disadvantage of this protocol is that this is a single path routing protocol so when an route is discovered and maintained, if the route is undiscovered then source cannot reach the destination.

3.2 Ad hoc on demand multipath distance vector routing protocol, AOMDV has been developed from a uni path ondemand routing protocol AODV. The key concept in AOMDV is computing multiple loop-free paths per route discovery. With multiple redundant paths available, the protocol switches routes to a different path when an earlier path fails. Thus a new route discovery is avoided. Route discovery is initiated only when all paths to a specific destination fail.

The AOMDV $[13,16]$ protocol finds multiple paths and this involves two stages which are as follows: i) A route update rule establishes and maintains multiple loop-free paths at each node, and ii) A distributed protocol finds link-disjoint paths. 
In [14], Thomas Clausen, Philippe Jacquet, Laurent Viennot both CBR traffic and TCP traffic, the AOMDV gives significant performance in RPGM model. Due to randomness in mobility among mobile nodes, the RWM model is widely used in MANETs and has selected AOMDV for evaluation due to its edge over other protocols.

In AOMDV [15] on the other hand is a multi-path routing protocol. It is an extension to AODV and also provides two main services i.e. route discovery and maintenance. Unlike AODV, every RREP is being considered by the source node and thus multiple paths discovered in one route discovery. Being the hop-by-hop routing protocol, the intermediate node maintains multiple path entries in their respective routing table. As an optimization measure, by default the difference between primary and an alternate path is equal to 1 hop. The route entry table at each node also contains a list of next hop along with the corresponding hop counts. Every node maintains an advertised hop count for the destination. Advertised hop count defined as the "Maximum hop count for all the paths". Route advertisements of the destination are sent using this hop count. An alternate path to the destination is accepted by a node if the hop count is less than the advertised hop count for the destination.

In AOMDV [17], describes that the changes are necessary in the basic AODV route discovery mechanism to enable computation of multiple link disjoint routes between source destination pairs. Note that any intermediate node _ on the route between a source _ and a destination _ can also form such multiple routes to _, thus making available a large number of routes between the network using the route discovery and the route maintenance. These authors have proved that the AOMDV performs better than the AODV protocol in an ad hoc nature.

In Ad-hoc On-demand Multipath Distance Vector (AOMDV) [19] proposed that Routing protocol is a multipath extension to the AODV protocol which maintains multiple loop-free and link disjoint paths. The routing entries for each destination contain a list of the next-hops and corresponding hop counts. Loop freedom is assured for a node by accepting alternate paths to destination if it has a less hop count than the advertised hop count for that destination. Because the maximum hop count is used, the advertised hop count therefore does not change for the same sequence number [18].

In [20] we provide a comparison of AOMDV and DSR-MP, two well known multi-path ad-hoc routing protocols. They specifically studied their performance in a wireless hybrid mesh network comprising of static MESH ROUTERs and dynamic MESH CLIENTs. Those results indicate both protocols, when assisted by MESH ROUTERs; have a high packet delivery rate even under excessive MESH CLIENT mobility. Compared to DSR-MP, AOMDV is able to establish more optimal paths at the cost of a higher routing overhead

\section{Advantages and Disadvantages of the AOMDV Routing Protocol}

AOMDV is affected more since it also has the additional overhead of more RREPs per route discovery .It is an multipath so that it requires an multiple paths from source to the destination when compared to AODV protocol The route maintenance mechanism of AOMDV requires known routes to be frequently purged. This results in a new route discovery for every new data connection but this is not found in AODV protocol

\subsection{Layered Cluster based routing protocol, LBCR Clustering}

Clustering is a process that divides the network into interconnected substructures, called clusters. Each cluster has a cluster head $(\mathrm{CH})$ as coordinator within the substructure. Each $\mathrm{CH}$ acts as a temporary base station within its zone or cluster and communicates with other CHs.

LCBRP (Cluster Based Routing Protocol) is an on-demand routing protocol, where the nodes are divided into clusters. It uses clustering's structure for routing protocol. Clustering is a process that divides the network into interconnected

Sub structures, called clusters. Each cluster has a cluster head as coordinator within the substructure. Each cluster head acts as a temporary base station within its zone or cluster and communicates with other cluster heads. Layered cluster is the process by the cluster that have the nodes in it partition themselves into layer.

In [21, 22], proposed "Layered Cluster-based Routing" (LCR) as a hierarchical reactive routing protocol for dense networks. This protocol uses a new concept, called "direction", which reduces flooding zone when discovering routes and thus decrease the number of exchanged control packets in the network. However, in some scenarios, this concept can prevent the RREQ packet from reaching the destination and consequently route establishment. The basic version of the introduced mechanism is suitable for almost fixed environment and topologies with DN node positioned at the network boundary. In this paper, we propose an enhanced version of LCR that can be used for all topologies under different conditions. Then we prove by simulations that LCR is well appropriate for dense and large sized Ad hoc networks.

In this paper,[23] the author has propose a new hybrid routing algorithm for MANET called Layered Cluster based Routing Protocol (LCRP) algorithm. It uses clustering's structure to decrease routing control overhead and improve the networks scalability. Results of our simulations show that the packet delivery ratio increases greatly and packet delay decreases significantly, when compared with other routing algorithms such as ad hoc on-demand Distance Vector (AODV).

Clustering management has five outstanding advantages over other protocols. First, it uses multiple channels effectively and improves system capacity greatly [23]. Second, it reduces the exchange overhead of control messages and strengthens node management [24].Third; it is very easy to implement the local synchronization of network [24, 25]. Fourth, it provides quality of service (QoS) routing for 
multimedia services efficiently. Finally, it can support the wireless networks with a large number of nodes.

\section{Advantages and Disadvantages of the LCR Routing Protocol}

LCR uses the layer cluster that inhibits Layered concept that improves node management, reduces control overhead and provides QOS parameters. The main disadvantage is that the selection of cluster head election that causes the control overhead. There are traffic bottleneck and single point failures at the cluster heads and gateways.

\section{Conclusion}

Based on the comparison of various clustering techniques for MANET and the discussion of this protocol in the previous section, we can conclude that the AOMDV works better than the other protocol because they have some more disadvantages when compared.

\section{References}

[1] C. E. Perkins and E. M. Royer, "Ad-hoc on demand distance vector routing," in Proceedings of the 2nd IEEE Workshop on Mobile Computing Systems and Applications (WMCSA'99), vol. 3, New Orleans, LA, USA, February 1999, pp. 90-100

[2] RFC3561: Ad hoc On-Demand Distance Vector (AODV) Routing.

[3] Mahesh K. Marina and Samir R. Das, "On-demand Multiple Distance Vector Routing in Ad Hoc Networks", Proceedings of the International Conference for Network Protocol, 2001.

[4] S. Bahk and M. E. Zarki. Dynamic Multi-path Routing and How it Compares with other Dynamic Routing Algorithms for High Speed Wide Area Networks. In Proceedings of the ACM SIGCOMM, pages 53-64, 1992.

[5] A. Bhatnagar and T. G. Robertazzi, "Layer Net: a new self-organizing network protocols,” Proc. IEEE MILCOM '90, pp. 845-849

[6] C. E. Perkins, E. RM. Royer, and Samir R. Das. "Ad Hoc On-Demand Distance Vector (AODV) Routing”, IETF MANET Working Group, Internet-Draft, March 2000.

[7] Zahian Ismail, Rosilah Hassan 2011. Effects of Packet Size on AODV Routing Protocol Implementation in Homogeneous and Heterogeneous MANET. In Proceedings of the IEEE Third International Conference on Computational Intelligence, Modelling \& Simulation

[8] Misra ,R.; Manda,C.R.; “ Performance Comparison of AODV/DSR On-Demand Routing Protocols for Ad Hoc Networks in Constrained Situation”, IEEE ICPWC 2005.

[9] Du, X. 2005. Designing efficient routing protocol heterogeneous sensor network. In proceeding of the 24th IEEE international Conference on Performance Computing and Communication.

[10] "Mobile Ad Hoc Networks and Routing Protocols", Handbook of Wireless Networks and Mobile Computing, Edited by Ivan Stojmenovic' Copyright (C)
2002 John Wiley \& Sons, Inc. ISBNs: 0-471- 41902-8 (Paper); 0-471- 22456-1 (Electronic).

[11] Zheniqiang Ye, Strikanth V. Krishnamurthy and Satish K. Tripathi, "A Framework for beliable Routing in Mobile Ad Hoc Networks”, IEEE INFOCOM, 2003

[12] DCd, "Link-state routing with hop-byhop forwarding can achieve optimal traffic engineering," in Proc. IEEE INFOCOM, Apr. 2008, pp. 466-474.

[13] V.C.Patil, Rajashree, V.Biradar, R.R. Mudholkar, S.R.Sawant, "On-Demand Multipath Routing Protocols for Mobile Ad Hoc Networks Issues and Comparison", International Journal of Wireless Communication and Simulation, 2010,Vol. 2(1), pp. 21-38.

[14] Thomas Clausen, Philippe Jacquet, Laurent Viennot, "Comparative study of CBR and TCP

[15] Performance of MANET routing protocols”, Technical report, Project HiPERCOM, INRIA Rocquencourt, 2002. Mahesh K.Marina and Samir R.Das,"Adhoc Multipath On Demand Distance Vector Routing", Published online in Wiley Inter science, 2006. Pages : 969-988.

[16] Asis Nasipuri , Robert Castarida, Samir R. Das," Performance of Multipath Routing for On- Demand Protocols in Mobile Ad Hoc Networks", Mobile Networks and Applications 6, 339-349, 2001 (C) 2001 Kluwer Academic Publishers. Manufactured in The Netherlands.

[17] S. Bahk and M. E. Zarki. Dynamic Multi-path Routing and How it Compares with other Dynamic Routing Algorithms for High Speed Wide Area Networks. In Proceedings of the ACM SIGCOMM, pages 53-64, 1992.

[18] H.D.Trung, W.Benjapolakul, P.M.Duc, "Performance evaluation and comparison of different ad hoc routing rotocols”, Department of Electrical Engineering, hulalongkorn University, Bangkok, Thailand, May 2007

[19] S. R. Biradar1, Koushik Majumder2, Subir Kumar Sarkar3, "Performance Evaluation and Comparison of AODV and AOMDV" (IJCSE) International Journal on Computer Science and Engineering Vol. 02, No. 02, 2010, 373-377

[20] I. F. Akyildiz and X. Wang, “A Survey on Wireless Mesh Networks,” IEEE Communications Magazine, vol. 43, no. 9, pp. S23-S30, 2005.

[21] I. Jemili, A. Belghith and M. Mosbah, "A layered cluster-based routing protocol for an Ad Hoc environment", in Proceedings of the ACS/IEEE

[22] International Conference on Computer Systems and Applications AICCSA'10, pp. 1-8, Washington DC, 2010.

[23] M. Jiang, J. Li, Y. C. Tay, "Cluster Based Routing Protocol", Internet draft. IETF MANET Working Group, August 1999.

[24] A. B. McDonald and T. F. Znati, “A mobility based framework for adaptive clustering in wireless ad hoc networks,” IEEE J. Select. Areas Commun., vol. 17, no. 8, Aug. 1999, pp. 1466-1487.

[25] C. R. Lin and M. Gerla, "Adaptive clustering for mobile wireless networks,” IEEE J. Select. Areas Commun., vol.15, no. 7, Sep. 1997, pp. 1265-1275. 


\section{Author Profile}

N.Nithya received the B.Sc. and M.Sc. degrees in Computer Science from Government Arts \& Science College, Valparai and Bharathiyar University, Coimbatore in 2009 and 2011 respectively. Currently doing Currently doing Master of Philosophy in Computer Science in Dr. SNS Rajalakshmi College of Arts \& Science, Coimbatore.

Mrs. R. Maruthaveni has completed her UG at Bharathithasan University and MCA at Madras University, and M.Phil at Periyar University. Now, she is working as an Assistant .Professor in Department of computer Science at Dr.SNS Rajalakshmi College of arts and Science, Chinnamettupalayam, Coimbatore- 35 . 